\title{
Analyzing discourse and identity in physics education: methodological considerations
}

\author{
Anders Johansson ${ }^{1,2}$ \\ ${ }^{1}$ Department of Physics and Astronomy, Uppsala University, Box 516, 75120 Uppsala, Sweden \\ ${ }^{2}$ Centre for Gender Research, Uppsala University, Box 527, 75120 Uppsala, Sweden
}

(Dated: September 19, 2016)

\begin{abstract}
Physics Education Research has for a long time primarily been concerned with helping students to learn physics and to "think like a physicist." This paper explores the emerging subfield where students' identity development is analyzed to examine processes of "becoming a physicist" in a wider sense. Drawing on sociocultural theories and methodologies, and specifically analyzing identity and discourse, I focus on what happens when students with differing outlooks on physics encounter advanced physics courses. A discourse analytical framework allows one to inquire into the messages about "who one should be as a physicist" communicated to students during courses. This enables a discussion of what physicist identities are made possible for physics students. In this way, a discourse perspective can be one way of analyzing identities in physics without taking the norms of the discipline for granted.
\end{abstract}

\section{INTRODUCTION}

What does it mean to become a physicist? Physics Education Research (PER) has from the beginning been concerned with students' understanding of physics concepts and problem solving, and later their (mostly cognitive) attitudes and beliefs about physics and learning [1]. Studies asking questions about belonging and identity have only recently become part of the PER literature, however. This kind of research shifts the primarily cognitive focus of PER to ask questions of a more social nature. Becoming a physicist does not only concern "learning to think like a physicist" [2], this strand of research argues, but also to act and talk like a physicist, and see oneself and be seen as a "physics person." All these aspects of learning are social and involve more than just the cognitive processes of individuals, hence this research draws on the wide "sociocultural approach" to learning [3].

Focusing on students' identity can be viewed as taking a "participation perspective" on physics, rather than a "content" or "discipline" perspective, since it often puts students" experiences of studying physics first. Specifically, it has been argued that identity studies are important for understanding diversity issues in education, such as persistence and drop-out, since the full sociocultural context of students' learning experiences are taken into account [4].

This article is a methodological reflection that discusses how PER researchers could gain an increased understanding of social processes in physics learning environments by taking inspiration from certain theories and methodologies developed in social research on identity. In particular, I will discuss the poststructuralist notion of discourse as a social structure for interpretation that shapes what can be said, done or understood in a certain context. Drawing on two studies from my PhD-project $[5,6]$ and other studies of identity in physics education $[4,7-10]$, I will argue that using a discursive perspective of identity enables us to answer important questions about students' various relationships to physics without taking the norms of the discipline for granted. For example, in the projects reported here, I and my co-authors have seen how students' negotiations of learning practice in an electromagnetism course can be affected by cultural norms around physics and how the teaching and learning practice in quantum physics classrooms can limit the ways of identifying as a physicist for students.

\section{IDENTITY AND DISCOURSE}

Studies of identity in PER and in the wider field of science education have used many different approaches to answer questions about the social processes of learning science. In this section, I will give an overview of the field and some of these approaches and theories, and discuss some of the critical issues still facing this kind of research.

\section{A. Identity studies in PER}

In the last 15 years, researchers in science education have increasingly started to explore the social aspects of science learning by using identity and related concepts. Similarly, a growing subfield of PER has started to ask questions about identity. This has been especially pronounced in relation to questions of gender equality or the participation of "minority students" in physics (e.g. [4, 8], see also the newly published "Focused Collection: Gender in Physics" in PRPER [11]).

The overall focus on identity in education research in recent years builds on a more or less social constructionist framework. Here, identity is not seen as a stable property belonging to a single person, but rather as a socially constructed position and sense of self that is acquired through socialization and learning, but also constantly negotiated in social interaction [12]. Much focus has been put on how students, with varying backgrounds, can be helped in acquiring "appropriate" "science identities" 
or "physics identities," which might be seen as one part of an individual's overall identity [4, 10, 13, 14].

Being seen as a socially constructed notion of "who someone is," built on socially negotiated meanings, identity is a concept which is hard to study in a purely quantitative way. Hence many studies discussing identity use a qualitative and interpretative research framework [15], although some attempts to operationalize identity in statistical analyses have been attempted [4]. The qualitative studies aim at gaining an understanding of the social world of research subjects, often to be able to explain how particular social contexts "work," rather than finding general patterns or laws [16].

Some PER studies on identity have focused on mapping out students" "physics identity" through large survey-based investigations [4, 17, 18]. In this body of work, "physics identity" is modeled as self-reported concepts of recognition, performance, comptence and interest. This model, built on earlier qualitative work [14], has been used in regression analyses to show that identifying as a "physics person" correlates with choosing physics, but also that certain factors seem to influence physics identity more than others [4, 17, 18]. A few gender differences have also been found [4]. Although giving a general picture of some dimensions of identity, these quantitative results will generally not be able to give nuanced pictures of the details of students' identity negotiations. This is recognized from the onset by the authors behind the quantitative physics identity model who claim that "in addition to focused experimental or quasi-experimental quantitative studies, explanatory qualitative and case-study work is a necessary follow-up" ([4], p. 998). A movement towards using more qualitative or mixed-methods approaches can be seen. For example, a later mixed-methods study involving one of the same authors investigate high school students' physics identity development through analyzing and comparing four teachers' positioning work and students' engagement in each of the teachers' classes [19].

Several studies employing a qualitative, ethnographic approach have used the communities of practice framework [20] to investigate students' enculturation into physics communities. These studies are often focused on upper-division courses or graduate work, and are aimed at examining students' possibilities of acquiring identities as physicists, that is, becoming part of a community of professional physicists [9, 10, 21]. One take-away message from these studies is that the notion of a physics expert, often invoked in PER research, needs to include social and cultural aspects apart from the cognitive aspects usually discussed [9]. A recent study of the identity development of undergraduate students in a Physics Learning Assistant program has used a combined framework of the previously discussed "physics identity" model [4] and communities of practice theory to bridge the gap between self-concept and practice in identity [8].

Lemke claims that an extensive familiarity with theories from social science should be preferred when applying sociocultural approaches to science education, and that this seldom has been the case [3]. In PER identity research, the two approaches mentioned above, physics identity as self-perception and communities of practice have been given the most attention. However, some studies of identity in physics education have brought in a wider variety of theoretical perspectives from social science.

The work of Danielsson and Gonsalves, recently summarized in a joint article [7], is a good example of this wider use of sociocultural theories. These authors draw on the communities of practice framework, but have in their studies of identity negotiations among undergraduate and graduate physics students integrated this with discourse analysis and theories from gender research such as "doing gender," performativity, and masculinities as a social construct. These frameworks allow the authors to identify how constructions of masculinity are intertwined in complex ways with ideas about the skills expected of a competent physicist. This means that being recognized as a competent physicist can be a process that involves specific negotiations of gender identity, e.g. female physicists repudiating femininity in order to be "one of the boys."

\section{B. Extending the view of identity}

The various approaches to studying identity in physics education have given many valuable results, which may help us to understand both the situations of students who are already clearly oriented towards physics and those who struggle with finding a place in physics. However, the scope of the discussions of identity could be widened by bringing in other perspectives and theories from social science. In particular, theories that focus on the reproduction of the culture of physics and its specific norms seem to be mostly absent from the current studies of identity in PER.

While the popular communities of practice framework may provide valuable insights into how students come to be part of different communities, the common use of it in science education has been criticized for making the question of identity in science primarily to be about individuals' agency, and not about the structures limiting that agency. In Shanahan's words, the communities of practice framework "tends to take the norms as given and attends to how individuals navigate those norms" ([13], p. 57). Hence, Shanahan calls for more studies that focus on the construction and reproduction of norms in science education contexts. Another similar problem with the communities of practice framework may be its limited scope when it comes to discussing power or gender [22].

Focusing on the construction and reproduction of norms or social structures means turning the analytical lens away from the individual and onto the cultural contexts which individuals have to navigate. This also puts into question some of the concepts used for discussing students' identities. For instance, when using the notion of "enculturation" we should always, in my view, ask: Enculturation into what culture? What are the norms of that culture? How are they reproduced and what does this mean for students? Similarly, the concept "identity development" perhaps implies that there is a certain "correct" path that development should follow, and that it is this path 
that we should lead all students along. That kind of implicit assumption, if present, needs to questioned. My view is that without discussing the norms of the discipline, research about identity development or enculturation may end up being highly normative in that it may address research questions only from the perspective of the discipline.

How can studies of identity in physics education that focus on the norms students encounter be designed? In the next section I will outline a discursive view of identity, inspired by poststructuralist theory, where identities are viewed as always negotiated in relation to existing subject positions in discourse. This approach is one way of including an analysis of power and social structures in identity research, to avoid focusing only on individuals' dealings with taken-for-granted structures.

\section{Using a discursive view of identity}

Poststructuralist use of the term discourse often builds on the work of Michel Foucault (for an overview, see Ref. [12]). In this tradition, discourse is seen as not just language, but as an overarching, dynamic, social structure, which defines what can be said, acted out and comprehended. In the socially constructed world of human consciousness, discourse limits our conceptualizations of the world [23]. Studying identities with a discursive perspective then means focusing on the recognition or non-recognition of actors in discourse [24, 25]. This can be done on different levels, emphasizing either the possibilities for individuals to express themselves as certain recognizable subjects or the broader structures of available or recognizable subject positions in discourse (see Ref. [23] for an overview of some approaches for analyzing discourse).

Most approaches to discourse analysis, in line with Foucault's work, view power as inherent in discourse. In defining who is an appropriate or recognizable subject, power is exerted through discourse. The struggle for definition in discourse has been described for instance as a fight over "social goods" [25] or a struggle for "hegemony" $[23,26]$. One way of applying these theories in a study of a specific context can be to map out the "dominant discourse" in that context and find out what subject positions are made available through that discourse. Some research in science education has used this approach to study for instance the possibilities for teen girls to identify as a science person $[27,28]$ or for biotechnology students to be recognized as proper biotechnologists [29]. This line of inquiry is the one that I have adopted in my studies.

\section{INSIGHTS FROM EMPLOYING A DISCURSIVE VIEW OF IDENTITY}

I will draw on two studies from my $\mathrm{PhD}$ research to exemplify how a discursive view of identity can produce new and valuable insights about physics education. These examples also demonstrate the value of qualitative and mixed-methods approaches for understanding students' experiences and ac- tions. In both these studies, I and my co-authors have used a discourse analytical framework inspired by Gee, who emphasizes how discourse "builds" several things in the world, like significance, practice, identity, relationships etc. [25]. The form of our discourse analysis has then been to ask what is built and not built in the discourse we analyze. What culture is reproduced and which identities are made possible or enacted?

In the first study, Andersson and I explore the situation of an apparent gender gap in grades in an undergraduate course in electromagnetism [5]. Although an initial quantitative comparison of grades seemed to show clear differences between men and women, we were able to elaborate the picture. We complemented the initial statistics with qualitative data from interviews with students who had finished the course and used this as a basis for our discourse analysis. The analysis focused on how practice, significance and identity were built in the discourse of the interview interactions.

The practice students adopted in the course was clearly related to how they viewed themselves, but to a larger extent in terms of program or vocational identity rather than gender identity. As one of our informants phrased it: "Electromagnetism seemed narrower than I had expected. I couldn't see the use of it in the fields I plan to work in." We interpret this as the course communicating certain norms about what physics is about, norms drawn from the discipline and culture of physics, the discipline of all instructors, which don't necessarily "match" students' identities. A discourse analytical framework was an essential tool for reaching those conclusions and getting beyond a dichotomous analysis of gender.

In the second study, I and my co-authors take a similar discursive approach to undergraduate quantum physics [6]. In this ethnographically inspired study of three courses in quantum physics at Swedish universities, we found that many different views of what quantum physics is about were expressed in the discourse of courses, teachers and students. It could be a start of doing "real" physics, a way of getting deeper into philosophical questions about the universe or a tool for technological applications. However, the dominant discourse in the courses was primarily constructing the doing of quantum physics as a practice of "calculating." While being able to solve standard, pen and paper problems is of course one big part of learning quantum physics, we argue that the extensive focus on this in courses limits the available positions for enacting a discursive identity as a "good" quantum physics student. A good quantum physics student is expected to be a good "calculator," but is at the same time implicitly expected to have a good grasp of the "big picture," something which puts students into a kind of double-bind.

We argue that the norms expressed through the discourse of quantum physics courses risk excluding people, in limiting the ways of being recognized as a physicist. At the same time, the discourse of these undergraduate courses may be seen as taking part in the reproduction of certain aspects of physics culture on a larger scale, that is, the ongoing establishment of certain norms about what physics "is." We find some of the norms reproduced by the courses to possibly promote a physics culture that is both instrumental (as it skims over philosophical 
and other questions) and elitist (as a "good student" is still expected to grasp more than what is given in class).

\section{CONCLUSIONS}

Both the studies described above point to how certain norms about physics are communicated in physics education, and how these norms may limit students' possibilities for learning physics or striving to become physicists. These results were found by using a discursive view of identities, and qualitative and mixed-methods research approaches. In this way I and my co-authors can, like much of the identity-orientated research in PER has done, identify many hurdles in students' development of physics identities. However, adopting a discursive approach can bring out students' encounters with the discourse of physics without taking the norms of the discipline for granted. This view involves a questioning of the idea of the acquisition of physics identities. If identity "development" is a question of enculturation into a culture, I find it important to ask what this culture entails.

I want to conceptualize this framework as a participationcentered view of physics education, where the situation students are put in is foregrounded, rather than the priorities of the discipline. This is not always the case in PER research, although some studies with this critical view have been pub- lished (e.g. [7, 19]). It has been argued that a large part of the "bias" that affects women negatively in physics is due to certain aspects of the physics culture [30]. This points to the importance of research approaches that allow us to direct a critical gaze at the cultural norms of physics to gain insights that could bring about a more gender equal and diverse physics.

To conclude, in this paper I have argued for the value of broadening inquiries in PER to include social issues of physics learning, and especially for doing this with a discourse and identity-lens and qualitative methods. This will allow for asking questions about what the social frames for becoming a physicist are, and in doing so, shifting the analytic focus from students' negotiations of already existing norms to the reproduction of norms in the culture of physics. If these perspectives are more widely adopted in PER, I believe that PER research will be better able to answer pressing questions about equality and participation in physics. In the end, this strand of research hopes to bring change into physics education and physics research environments so that physics can be a place where a wider diversity of people can feel at home.

\section{ACKNOWLEDGMENTS}

Thanks to my supervisors and co-authors, Staffan Andersson, Maja Elmgren, and Minna Salminen-Karlsson.
[1] J. L. Docktor and J. P. Mestre, Phys. Rev. ST Phys. Educ. Res 10, 020119 (2014).

[2] A. Van Heuvelen, Am. J. Phys. 59, 891 (1991).

[3] J. L. Lemke, J. Res. Sci. Teach. 38, 296 (2001).

[4] Z. Hazari, G. Sonnert, P. M. Sadler, and M.-C. Shanahan, J. Res. Sci. Teach. 47, 978 (2010).

[5] S. Andersson and A. Johansson, Phys. Rev. Phys. Educ. Res. 12, 020112 (2016).

[6] A. Johansson, S. Andersson, M. Salminen-Karlsson, and M. Elmgren, Cult Stud of Sci Educ (2016), 10.1007/s11422016-9742-8.

[7] A. J. Gonsalves, A. Danielsson, and H. Pettersson, Phys. Rev. Phys. Educ. Res. 12, 020120 (2016).

[8] E. W. Close, J. Conn, and H. G. Close, Phys. Rev. Phys. Educ. Res. 12, 010109 (2016).

[9] I. Rodriguez, R. M. Goertzen, E. Brewe, and L. H. Kramer, Phys. Rev. ST Phys. Educ. Res. 11, 010116 (2015).

[10] P. W. Irving and E. C. Sayre, Phys. Rev. ST Phys. Educ. Res. 11, 020120 (2015).

[11] E. Brewe and V. Sawtelle, Phys. Rev. Phys. Educ. Res. 12, 020001 (2016).

[12] V. Burr, Social Constructionism (Routledge, London, 2003).

[13] M.-C. Shanahan, Stud. Sci. Educ. 45, 43 (2009).

[14] H. B. Carlone and A. Johnson, J. Res. Sci. Teach. 44, 1187 (2007).

[15] D. F. Treagust, M. Won, and R. Duit, in Handbook of Research on Science Education, Vol. Volume II, edited by N. G. Lederman and S. K. Abell (Routledge, 2014) pp. 3-17.

[16] V. K. Otero and D. Harlow, in Getting Started in PER, Re- views in PER No. 2, edited by C. Henderson and K. A. Harper (American Association of Physics Teachers, College park, MD, 2009).

[17] R. M. Lock, Z. Hazari, and G. Potvin, AIP Conf. Proc. 1513, 262 (2013)

[18] Z. Hazari, G. Potvin, R. M. Lock, F. Lung, G. Sonnert, and P. M. Sadler, Phys. Rev. ST Phys. Educ. Res. 9, 020115 (2013).

[19] Z. Hazari, C. Cass, and C. Beattie, J Res Sci Teach 52, 735 (2015).

[20] J. Lave and E. Wenger, Situated Learning: Legitimate Peripheral Participation (Cambridge University Press, 1991).

[21] P. W. Irving and E. C. Sayre, Phys. Rev. ST Phys. Educ. Res 10, 010109 (2014).

[22] M. Salminen-Karlsson, Scand J Manag 22, 31 (2006).

[23] M. W. Jorgensen and L. J. Phillips, Discourse Analysis as Theory and Method (SAGE Publications Ltd, London; Thousand Oaks, Calif, 2002).

[24] J. P. Gee, Rev. Res. Educ. 25, 99 (2000).

[25] J. P. Gee, An Introduction to Discourse Analysis: Theory and Method, 3rd ed. (Routledge, New York, 2011).

[26] E. Laclau and C. Mouffe, Hegemony \& Socialist Strategy (Verso, London, 1985).

[27] L. Archer, J. DeWitt, J. Osborne, J. Dillon, B. Willis, and B. Wong, Sci. Ed. 96, 967 (2012).

[28] S. Olitsky, Cult Stud of Sci Educ 1, 745 (2006).

[29] A. Solli, F. Bach, and B. Åkerman, Cult Stud of Sci Educ 9, 1 (2014).

[30] Z. Hazari and G. Potvin, Electron. J. Sci. Educ. 10 (2005). 